\title{
Assessment of the durability of robot-assisted laparoscopic sacrocolpopexy for treatment of vaginal vault prolapse
}

\author{
Daniel S. Elliott · Sameer A. Siddiqui · George K. Chow
}

Received: 1 November 2006 / Accepted: 24 May 2007 / Published online: 19 June 2007

(c) Springer London 2007

\begin{abstract}
Transabdominal sacrocolpopexy has been shown, in multiple long-term studies of its success and durability, to be the definitive treatment option for posthysterectomy vaginal vault prolapse. It is, however, associated with greater morbidity than vaginal repair. We describe a minimally invasive technique for vaginal vault prolapse repair and present our experience with a minimum of one-year follow-up. The surgical technique involves five laparoscopic ports-three for the da Vinci robot and two for the assistant. After appropriate dissection a polypropylene mesh is attached to the sacral promontory and to the vaginal apex by use of Gore-Tex sutures. The mesh material is then covered by the peritoneum. Patient analysis focused on complications, urinary continence, patient satisfaction, and morbidity, with a minimum of 12 months follow-up. Forty-two patients with post-hysterectomy vaginal vault prolapse underwent robot-assisted laparoscopic sacrocolpopexy at our institute and 35 have a minimum of 12 months follow-up, with a mean follow-up of 36 months (range 12-48) in the group. Mean age was 67 (47-83) years and mean operating time was $3.1(2.15-4.75) \mathrm{h}$ for the entire cohort. All but one patient were discharged home on postoperative day one; one patient left on postoperative day two. One developed recurrent grade three rectocele, one had recurrent vault prolapse, and two suffered from vaginal extrusion of mesh. All patients were satisfied with their outcome. The robot-assisted laparoscopic sacrocolpopexy is a minimally invasive technique for vaginal vault prolapse repair, combining the advantages of open sacrocolpopexy
\end{abstract}

D. S. Elliott $(\varangle) \cdot$ S. A. Siddiqui · G. K. Chow Department of Urology, Section of Female Urology and Laparoscopy, Mayo Clinic, 200 First Street S.W., Rochester, MN 55905, USA

e-mail: Elliott.daniel@mayo.edu with the reduced morbidity of laparoscopy. We observed reduced hospital stay, low occurrence of complications, and high patient satisfaction, with a minimum of 1-year followup. Most importantly, the long-term results of the robotic repair are similar to those of open repair, but with significantly less morbidity.

Keywords Robotics - Laparoscopy · Sacrocolpopexy · Vaginal vault prolapse

\section{Introduction}

More than one in nine women will undergo a hysterectomy and up to $10 \%$ of these will need surgical repair for treatment of a symptomatic vaginal prolapse [1,2]. The search for the ideal repair with the best combination of efficacy, safety, and durability for treatment of vaginal vault prolapse is an ongoing process, as evidenced by the multiple surgical approaches to this problem. Clearly, no surgical approach is ideal for every patient. As known risk factors for prolapse, for example age, obesity, and hysterectomy, continue to increase in the United States, however, so does the search for better means to repair vaginal vault prolapse [2-4].

In multiple studies transabdominal sacrocolpopexy has been shown to have the highest long-term success for durable repair of severe vault prolapse (93-100\%) [5-11]. In addition to high success and durable results, other advantages of the sacrocolpopexy approach with use of synthetic material to repair vault prolapse can be summarized as follows:

1. Support of the vaginal vault by the anterior surface of the sacrum preserves the normal axis of the vagina. 
2. Maximum vaginal depth is preserved, which is important to patients who desire continued sexual activity and to patients with an already foreshortened vagina from previous surgery.

3. Use of synthetic suspensory material can provide a source of strength in patients where the native tissue with prolapse is weak [5].

Young patients and those with an active life-style are usually ideal candidates for the open procedures. Other important indications are concurrent medical conditions such as chronic cough, COPD, and asthma, which place chronic and repeated increased intra-abdominal pressure on the repair. Unfortunately, because of the morbidity of the open transabdominal procedure, many patients are unable to tolerate the surgery and are, therefore, managed via a transvaginal approach or with more conservative therapies, for example expectant management, pessary, or even colpocleisis.

Clearly, the objectives of any surgical repair of vaginal vault prolapse should include restoration of proper anatomy, maintenance of function (sexual, bowel, and urinary), and durability. Surgical approaches to correct the prolapse include either vaginal or abdominal approach or a combination. The main advantage of the vaginal approach has historically been reduced morbidity, including shorter hospitalization and convalescence [12, 13]. Unfortunately, the long-term success of transvaginal repairs is consistently lower than for abdominal sacrocolpopexy [14].

In an effort to balance the benefit of open sacrocolpopexy with the advantage of vaginal repair, many attempts have been made to treat vault prolapse by laparoscopic sacrocolpopexy [15-18]. Unfortunately, technical difficulties and the potentially significant increase in operating time has greatly limited its widespread use. Recent advances in robotic surgery have helped to address the limitations of laparoscopy.

Robotics provides technical features such as threedimensional vision, increased robotic instrument maneuverability, and filtering of physiological tremor [19]. These factors provide an ergonomic environment for the surgeon and simplify complex laparoscopic tasks. We describe the technique of robot-assisted laparoscopic sacrocolpopexy using a polypropylene graft. Preliminary results of our approach have been reported elsewhere [20, 21]. Our objectives in this study were twofold:

1. To analyze the long-term durability of robot-assisted laparoscopic sacrocolpopexy on the basis of postoperative physical examination.

2. To determine the morbidity (postoperative pain, complications, and length of hospital stay) of the procedure compared to that associated with transabdominal procedures.

\section{Materials and methods}

After Institutional Review Board approval, 42 consecutive patients with post-hysterectomy vaginal vault prolapse were treated by robot-assisted laparoscopic sacrocolpopexy at the Mayo Clinic, Department of Urology, starting in September 2002. To meet selection criteria patient had to have a Grade 4/4 Baden Walker apical prolapse. Patients with multiple previous abdominal surgery or multiple other vaginal defects were excluded from the procedure. All patients were evaluated preoperatively with a history, physical examination, and post void residual check. Surgical options and informed consent were obtained in the standard fashion. The surgical procedure was conducted by a team comprising a fellowship-trained laparoscopic surgeon and a female urologist.

\section{Surgical technique}

The daVinci robot is an integrated computer-based system consisting of two interactive robotic arms, a camera arm, and a remote control with three-dimensional vision capability. It uses instruments with $6^{\circ}$ of freedom, providing the same flexibility as the human wrist. The working robotic arms are attached to reusable 8-mm trocars and the camera is placed through a standard $12 \mathrm{~mm}$ laparoscopic port. For optimum collision-free robot function, the angle created by the camera port and each working robotic port should be obtuse and the distance between the ports at least one handbreadth. In robotic surgery the motions of the surgeon at the remote-control unit are replicated by the robotic arms placed within the patient. During robotic surgery an assistant surgeon is scrubbed at the operating table. The assistant performs a variety of important robot-related tasks including alignment and exchange of instruments on the robotic arms. The assistant also performs operative maneuvers with conventional instruments, including tissue countertraction, hemostasis, hemoclip application, suction, and assistance during suturing.

For the procedure the patient is placed on the operating table in the dorsal lithotomy position. After general anesthesia is established a nasogastric tube is placed and both arms are tucked beside the torso. The patient is prepped from the nipples to proximal thigh including the vagina.

After abdominal insufflation using a Veress needle, we place a periumbilical Visiport optical trocar (AutoSuture, Norwalk, CT, USA) under direct vision to avoid visceral or vascular injury. Two standard laparoscopic ports for retraction are next introduced under direct vision-one 10-mm port right subcostal lateral to the rectus and one $5 \mathrm{~mm}$ port one hands-breadth inferior-laterally (Fig. 1). Next, two $8 \mathrm{~mm}$ robotic ports are placed lateral to the rectus two fingerbreadths superior to the iliac crest. 


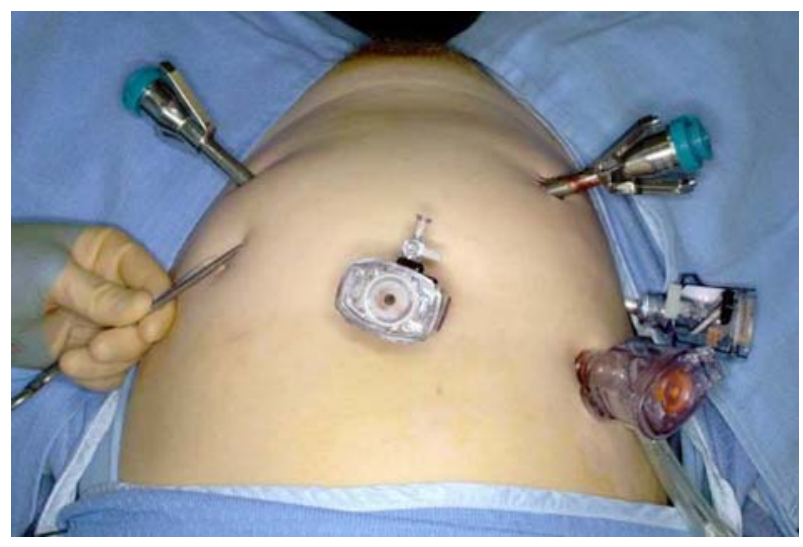

Fig. 1 Standard laparoscopic port placement for the robot-assisted laparoscopic sacrocolpopexy. The laparoscopic ports are the $10 \mathrm{~mm}$ right subcostal port and the $5 \mathrm{~mm}$ port inferior laterally. The robotic ports are the paraumbilical camera port and the two 8-mm working ports lateral to the rectus muscle superior to the iliac crest

Using standard laparoscopy, a retracting suture is placed through the sigmoid tenia to help in exposing the sacral promontory. The bladder is then dissected from the anterior vaginal wall using forceps and cautery scissors. A customized hand-held vaginal retractor manufactured at the Mayo Clinic (Fig. 2) is used to facilitate the dissection, which should be a relatively bloodless plane. Posteriorly, the peritoneal reflection is then incised to mobilize the vagina. Rectovaginal dissection is greatly facilitated by the vaginal

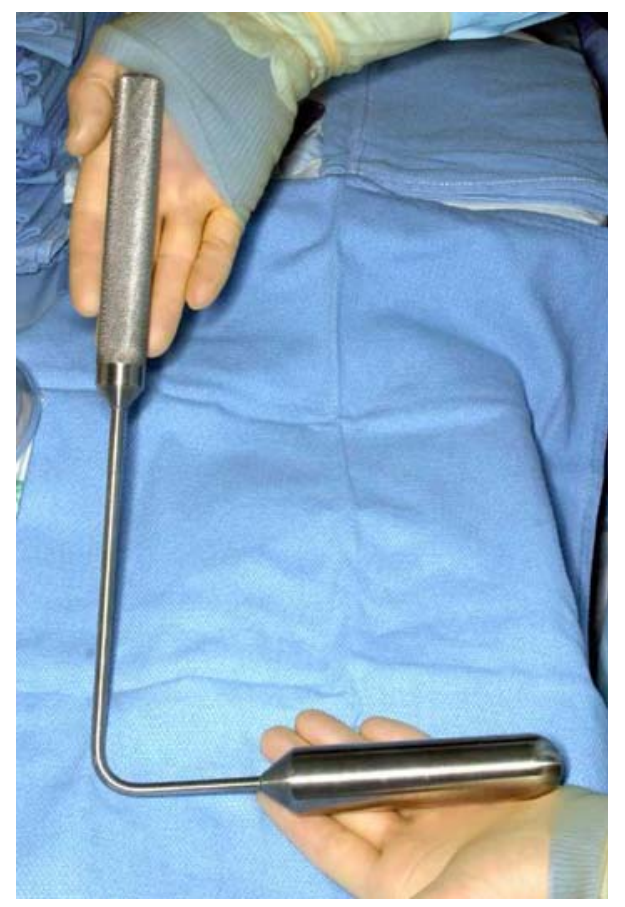

Fig. 2 The hand-held vaginal retractor (patent pending) used to facilitate dissection between the bladder and vagina retractor. Both of these dissections should be performed as distal (toward the introitus) as possible to maximize the support given by the Y-graft. After vaginal mobilization the sacral dissection is accomplished with avoidance of the sacral venous complexes. When the periosteum is exposed, the polypropylene Y-graft (IntePro American Medical Systems, Minnetonka, MN,USA) (Fig. 3) is brought into the field through the $10 \mathrm{~mm}$ port. In our experience the aforementioned steps are usually accomplished within $30-40 \mathrm{~min}$.

The robot is then docked with the base positioned at the foot of the bed. The robot is used to facilitate and greatly reduce the operating time needed for suturing of the graft to the vagina and the sacrum. The Y-graft is robotically sutured using 1.0 Gore-Tex. The $30^{\circ}$ lens and vaginal retractor maximize exposure for placement of the sutures. We have found placing the more difficult posterior sutures first followed by suturing the anterior portion of the Y-graft reduces the difficulty of the process. The tail of the graft is then sutured to the sacral promontory in an interrupted fashion with careful attention to avoiding tension on the vagina. Usually, three to five 1.0 Gore-Tex sutures are used to adequately fix the mesh to the sacrum. It is our preference to perform a standard Halban's culdoplasty with plication of the uterosacral ligaments at this time. The posterior peritoneum is then closed to completely retroperitonealize the graft [20, 21]. Postoperatively, pain is managed with scheduled Toradol 15-30 mg every $6 \mathrm{~h}$. Antibiotics are continued for two days postoperatively. Diet is advanced immediately and the patient is encouraged to ambulate on the evening of the procedure. All patients were discharged home on three months of vaginal estrogen postoperative, irrespective of preoperative estrogen status. The patients were followed with routine clinic visits and physical examination every three months for year.

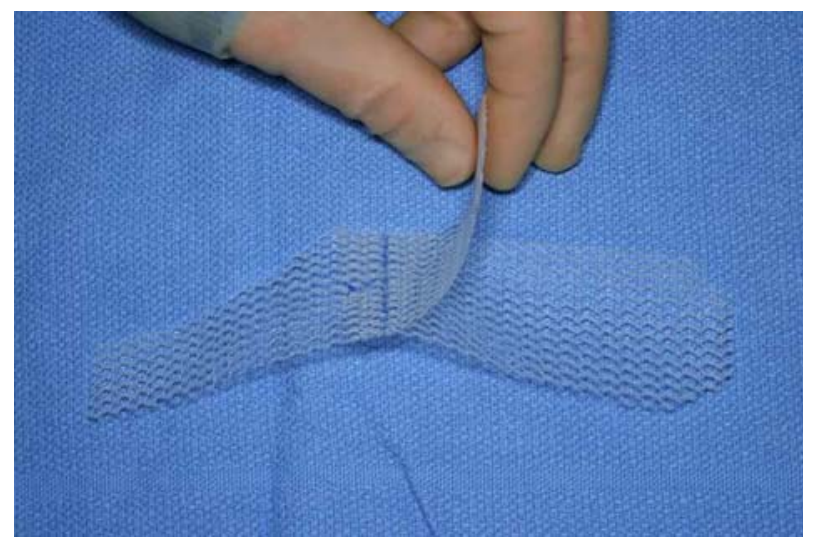

Fig. 3 The polypropylene Y-graft is used to tack the vaginal vault to the sacral promontory 


\section{Results}

At our institute we have performed robot-assisted laparoscopic sacrocolpopexy on 42 patients for treatment of grade 4/4 Baden Walker, symptomatic vaginal vault prolapse. Mean age is 67 (47-83) years. Thirty-five of these patients have met the minimum requirement of at least one-year follow-up (mean follow-up 24 months, range 12-48 months). Mean total operating time was 3.1 (2.15-4.75) hours. Figure 4 summarizes the operating time. Initially, the "skinto-skin" time was $4.75 \mathrm{~h}$. With experience, however, and use of several different time-saving steps we are now routinely completing the procedure in less than $2.5 \mathrm{~h}$. Of the 42 patients 19 had procedures less than $3 \mathrm{~h}$ and $5 \mathrm{had}$ procedures less than $2.5 \mathrm{~h}$.

Twenty-six $(61 \%)$ of the patients underwent a concurrent anti-incontinence procedure at the time of prolapse repair. These procedures included one cadaveric pubovaginal sling, twenty-two synthetic pubovaginal slings, and four transobturator slings. The anti-incontinence procedure was performed concurrently with the sacrocolpopexy and did not, therefore, lengthen the operating time.

Two patients were converted to an open procedure secondary to unfavorable anatomy. In both of these patients the bladder was densely adherent to the vagina from a previous anterior colporrhaphy and laparoscopic dissection was not possible. The open procedures were completed without difficulty.

All but one patient treated with the robotic sacrocolpopexy were discharged from the hospital after an overnight stay; one patient left on postoperative day two. All patients were dismissed on oral pain medication. Ten patients reported they only required non-steroidal

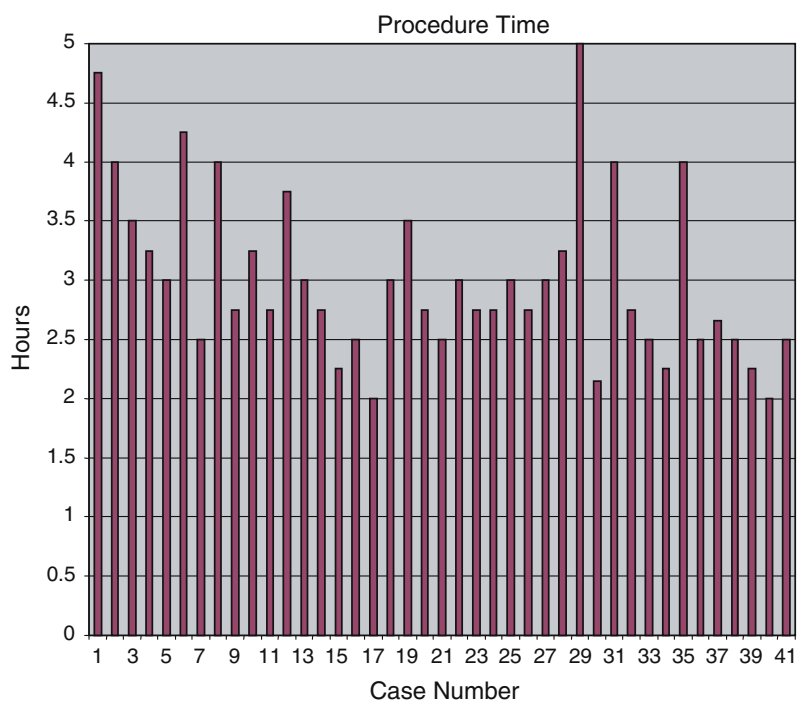

Fig. 4 Comparative operating time of robot-assisted laparoscopic sacrocolpopexy anti-inflammatory medication for control of their pain. One patient had persistent vaginal bleeding for 2 days post-operation, a complication related to the anti-incontinence portion of the procedure, because all bleeding was from the vaginal incision site. Although her hemoglobin remained stable with no further sequelae, this did require her to stay an extra night in the hospital.

All patients treated with the robotic sacrocolpopexy reported being satisfied with the outcome of their surgery and $41 / 42$ would recommend it to a friend. The one patient who did not recommend the procedure was the one converted to an open procedure.

\section{Complications}

\section{Immediate postoperative complication}

Complications were limited to mild port site infections in two patients, which resolved with oral antibiotic therapy.

\section{Long-term complications}

With a mean follow up of 36 months (range 12-48), 34/35 (97\%) have had a successful repair of their apical prolapse, on the basis of visual speculum examination one year postoperation. Examinations were performed by one of the authors (DSE). One patient, seven months after surgery, developed a recurrent vault prolapse. We elected to treat her recurrence with a transabdominal sacrocolpopexy. At the time of re-operation it was found the mesh was firmly attached to the sacrum but had completely separated from the vagina. Sutures were still attached to the mesh indicating the sutures had pulled through the vaginal mucosa. This was the fifth patient in our series leading us to adjust our suturing technique by adding two to four more sutures attaching the mesh to the vagina. Since this adjustment we have had no further recurrences, including those with less than one-year follow-up.

Another patient developed a recurrent grade 3/4 Baden Walker rectocele, without evidence of anterior or apical prolapse. This was the second patient in our series. The rectocele developed nine months after the sacrocolpopexy and was treated with an outpatient posterior colporrhaphy. It is now 36 months since the patient's posterior colporrhaphy and 47 months since sacrocolpopexy and she has not experienced any further recurrence of prolapse. As a result of this case we modified our robotic repair by placing the posterior leaf of the $Y$ graft to cover the entire vaginal length. Great effort is taken to place the mesh on the posterior aspect of the vagina as close to the level of the introitus as possible. Since this modification no patient in our series has experienced prolapse recurrence. 
Two patients developed a small $(1 \mathrm{~cm} \times 1 \mathrm{~cm})$ vaginal extrusion of the mesh at the level of the vaginal cuff. Each extrusion developed at 6 months after the procedure. The extrusion was managed as an outpatient with transvaginal excision of the mesh and primary closure. None experienced recurrence of their prolapse. These two patients were among our initial eight patients treated. We surmise that because all patients were treated with postoperative vaginal estrogen, overly aggressive dissection of the bladder off the vagina most probably contributed to this complication. Since modifying our dissection technique by avoiding excessive tension with our vaginal retractor and avoiding dissecting the vagina too thinly, no further patient has experienced this complication. Significant postoperative incontinence ( $>1 \mathrm{pad} /$ day) was experienced by two patients.

\section{Discussion}

The transabdominal sacrocolpopexy is a durable and effective treatment of post-hysterectomy vaginal vault prolapse. Not every patient is a candidate for this procedure, however, because of age, concurrent medical conditions, or concerns about postoperative recovery time. We feel the robot-assisted laparoscopic sacrocolpopexy accomplishes a repair identical with that of the open transabdominal technique. The morbidity associated with the open procedure is greatly reduced and the hospital stay has been reduced from 2 to 5 days with the open procedure to 1 day with the laparoscopic repair [22]. Also, on the basis of a minimum of one-year follow-up (mean 36 months), our results are as durable as the long-term results of the open procedure. Potentially, many more women will be offered the strongest repair for prolapse while still minimizing morbidity.

Relative contraindications would be the same for most laparoscopic procedures including patients with prior abdominal surgeries and those with morbid obesity. Clearly, longer follow-up is needed; the robot-assisted laparoscopic sacrocolpopexy described in this report may, however, be an ideal approach to surgical repair of vaginal vault prolapse.

Last, it must be stressed that we take great measures to perform the robot-assisted laparoscopic sacrocolpopexy to mimic the classic repair of the open transabdominal sacrocolpopexy including the Halban's culdoplasty. Recent reports have described laparoscopic "sacrocolpopexy" but on close review it becomes evident that even though highly skilled laparoscopic techniques were utilized, unfortunately, the basic principles of prolapse repair were not followed. Caution must be taken, because vaginal vault prolapse repair is not just a procedure of laparoscopic skills.
We do recognize some limitations of this study. It is a single surgical team, single institute experience and therefore the exact results may not be translatable to other institutes. Because this is a newly developed technique, time will be required to determine other institutes' success rates. This study was also of a relatively small cohort of patients. Despite these limitations, the study provides supporting evidence for durable long-term success and minimum complications of this minimally invasive procedure for treatment of pelvic organ prolapse.

\section{Conclusion}

We report the largest single institute series of robot-assisted laparoscopic sacrocolpopexy for treatment of high-grade vaginal vault prolapse. This minimally invasive technique provides the durable repair benefits of the open procedure with the minimal morbidity associated with laparoscopic surgery. Our series was characterized by a short inpatient hospital stay, minimal recurrence and conversion rates, minimal complications, and high overall patient satisfaction. The initial benefits associated with this novel procedure are promising and we expect more widespread use of robot-assisted laparoscopic sacrocolpopexy in the future.

Acknowledgments Authorship and contribution to this manuscript is limited to the three authors indicated. There was neither outside funding nor technical assistance with the production of this manuscript.

\section{References}

1. Marchionni M, Bracco GL, Checcucci V, Carabaneanu A, Coccia EM, Mecacci $F$ et al (1999) True incidence of vaginal vault prolapse. Thirteen years of experience. J Reprod Med 44:679

2. Olsen A, Smith V, Bergstrom J, Colling J, Clark A (1997) Epidemiology of surgically managed pelvic organ prolapse and urinary incontinence. Obstet Gynecol 89:501

3. Dwyer PL, Lee ETC, Hay DM (1988) Obesity and urinary incontinence in women. Br J Obstet Gynaecol 95:91

4. Virtanen HS, Makinen JI (1993) Retrospective analysis of 711 patients operated on for pelvic relaxation in 1983-1989. Int J Gynecol Obstet 42:109

5. Addison WA, Timmons MC (1993) Abdominal approach to vaginal eversion. Clin Obstet Gynecol 36:995

6. Timmons MC, Addison WA, Addison SB, Cavenar MG (1992) Abdominal sacral colpopexy in 163 women with posthysterectomy vaginal vault prolapse and enterocele. J Repro Med 37:323

7. Cundiff GW, Harris RL, Coates K, Low VHS, Bump RC, Addison WA (1997) Abdominal sacral colpoperineopexy: a new approach for correction of posterior compartment defects and perineal descent associated with vaginal vault prolapse. Am J Obstet Gynecol 177:1345

8. Snyder TE, Krantz KE (1991) Abdominal-retroperitoneal sacral colpopexy for the correction of vaginal prolapse. Obstet Gynecol 77:944 
9. Menefee SA, Miller KF, Wall LL (1999) Results of abdominal sacral colpopexy using polyester mesh in the treatment of posthysterectomy vaginal vault prolapse and enterocele. Obstet Gynecol 54:563

10. Reddy K, Malik TG. (2002) Short-term and long-term follow-up of abdominal sacrocolpopexy for vaginal vault prolapse: initial experience in a district general hospital. J Obstet Gynaecol. 22:532

11. Webb MJ, Aronson MP, Ferguson LK, Lee RA (1998) Posthysterectomy vaginal vault prolapse: primary repair in 693 patients. Obstet Gynecol 92:281

12. Podratz LK, Ferguson VR, Lee RA, Symmonds RE (1995) Abdominal sacral colpopexy for posthysterectomy vaginal vault descensus. Obstet Gynecol 50:719

13. Karram M, Goldwasser S, Kleeman S, Steele A, Vassallo B, Walsh P (2001) High uterosacral vaginal vault suspension with fascial reconstruction for vaginal repair of enterocele and vaginal vault prolapse. Am J Obstet Gynecol 185:1339

14. Benson JT, Lucente V, McClellan E (1996) Vaginal versus abdominal reconstructive surgery for the treatment of pelvic support defects: a prospective randomized study with long-term outcome evaluation. Am J Obstet Gynecol 175:418

15. Ostrzenski A (1996) Laparoscopic colposuspension for total vaginal prolapse. Int J Gynaecol Obstet 55:147
16. Cosson M, Rajabally R, Bogaert E, Querleu D, Crepin G (2002) Laparoscopic sacrocolpopexy, hysterectomy, and burch colposuspension: feasibility and short-term complications of 77 procedures. J Soc Laparoendosc Surg 6:115

17. Paraiso MF, Walters MD, Rackley RR, Melek S, Hugney C (2005) Laparoscopic and abdominal sacral colpopexies: a comparative cohort study. Am J Obstet Gynecol 192:1752

18. Higgs PJ, Chua HL, Smith AR (2005) Long term review of laparoscopic sacrocolpopexy. BJOG 112:1134

19. Hubens G, Coveliers H, Balliu L, Ruppert M, Vaneerdweg W (2003) A performance study comparing manual and robotically assisted laparoscopic surgery using the da Vinci system. Surg Endosc 17:1595

20. DiMarco DS, Chow GC, Gettman MT, Elliott DS (2004) Roboticassisted laparoscopic sacrocolpopexy for treatment of vaginal vault prolapse. Urology 63:373

21. Elliott DS, Krambeck AE, Chow GK (2006) Long-term results of robotic assisted laparoscopic sacrocolpopexy for the treatment of high grade vaginal vault prolapse. J Urol 176:655

22. Cosson M, Bogaert E, Narducci F, Querleu D, Crepin G (2000) Laparoscopic sacral colpopexy: short-term results and complications in 83 patients. J Gynecol Obstet Biol Reprod (Paris) 29:746 